Supporting Information for

\title{
Interfacial vs. Bulk Phenomena Effects on the Surface Tensions of Aqueous Magnetic Surfactants in Uniform Magnetic Fields
}

Derek M. Reed ${ }^{a}$, Alex Fortenberry ${ }^{a}$, Emily A. Wolfe $^{a}$, Rachel A. Stanhope ${ }^{a}$, Carla I. Daniel ${ }^{b}$, Caleb M. Hern ${ }^{a}$, Adam E. Smith ${ }^{a}$, Paul Scovazzo ${ }^{a *}$

${ }^{\text {a }}$ University of Mississippi, Department of Chemical Engineering Oxford, MS 38655

b LAQV/Requimte, Departamento de Química, Faculdade de Ciências e Tecnologia, Universidade Nova de Lisboa, Campus de Caparica, 2829-516 Caparica, Portugal

* Correspondence Email: scovazzo@olemiss.edu

Number of Pages (excluding cover sheet): Four

Number of Figures: Four

Number of Tables: Three 


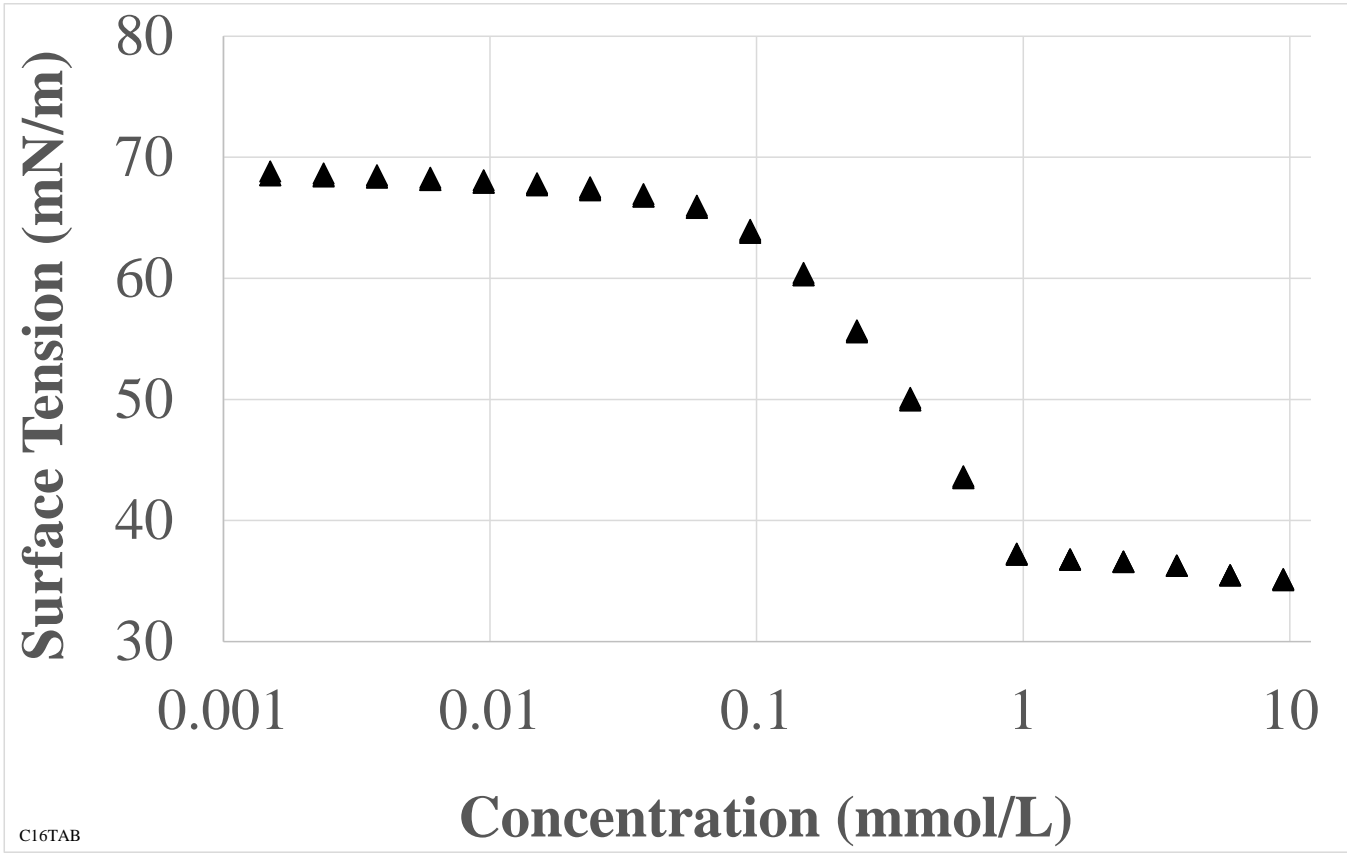

Figure S1. Tensiometer data for $\mathrm{C}_{16} \mathrm{TAB}$ used to determine the critical micelle concentration at $0.95 \mathrm{mmol} / \mathrm{L}$.

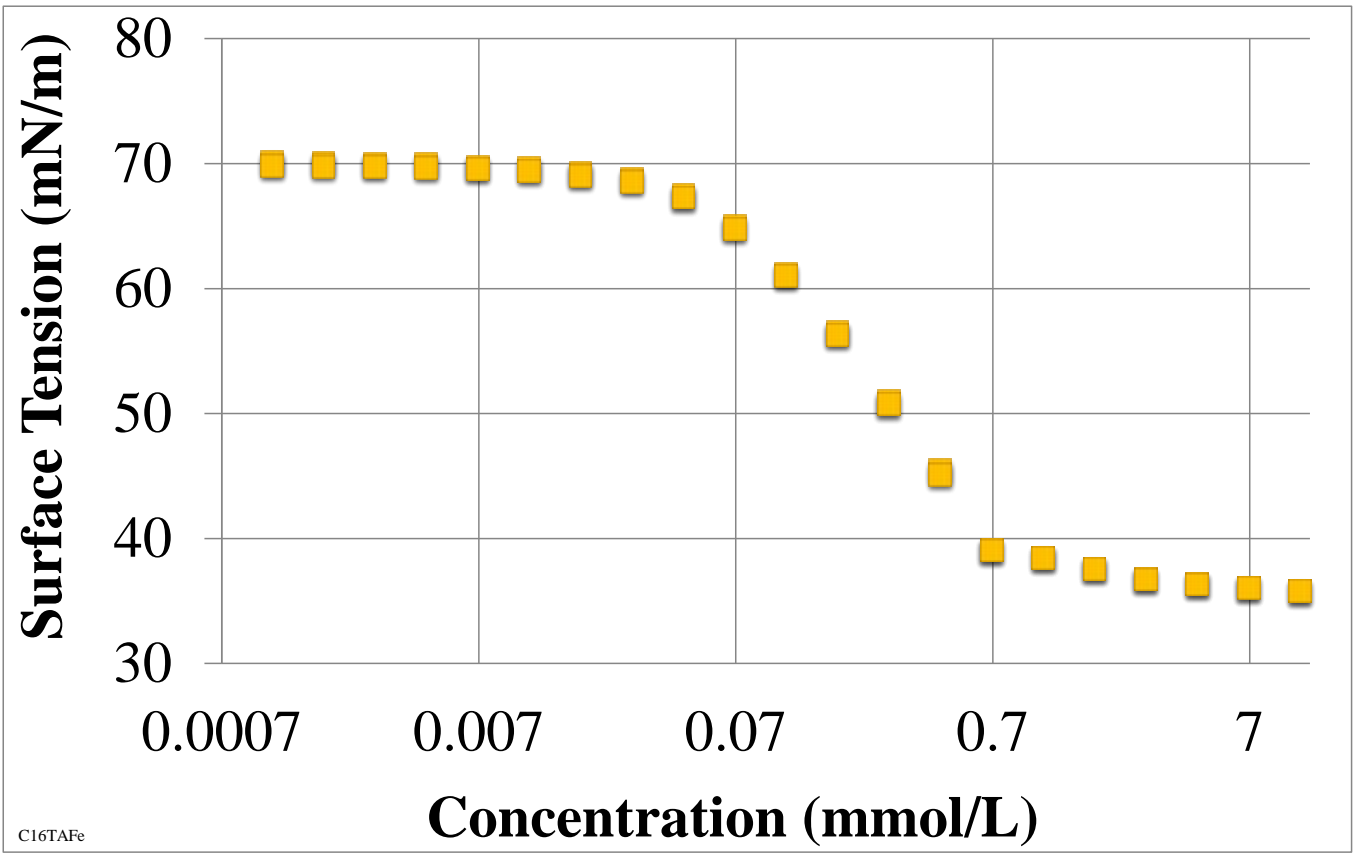

Figure S2. Tensiometer data for $\mathrm{C}_{16} \mathrm{TAFe}$ used to determine the critical micelle concentration at $0.69 \mathrm{mmol} / \mathrm{L}$. 


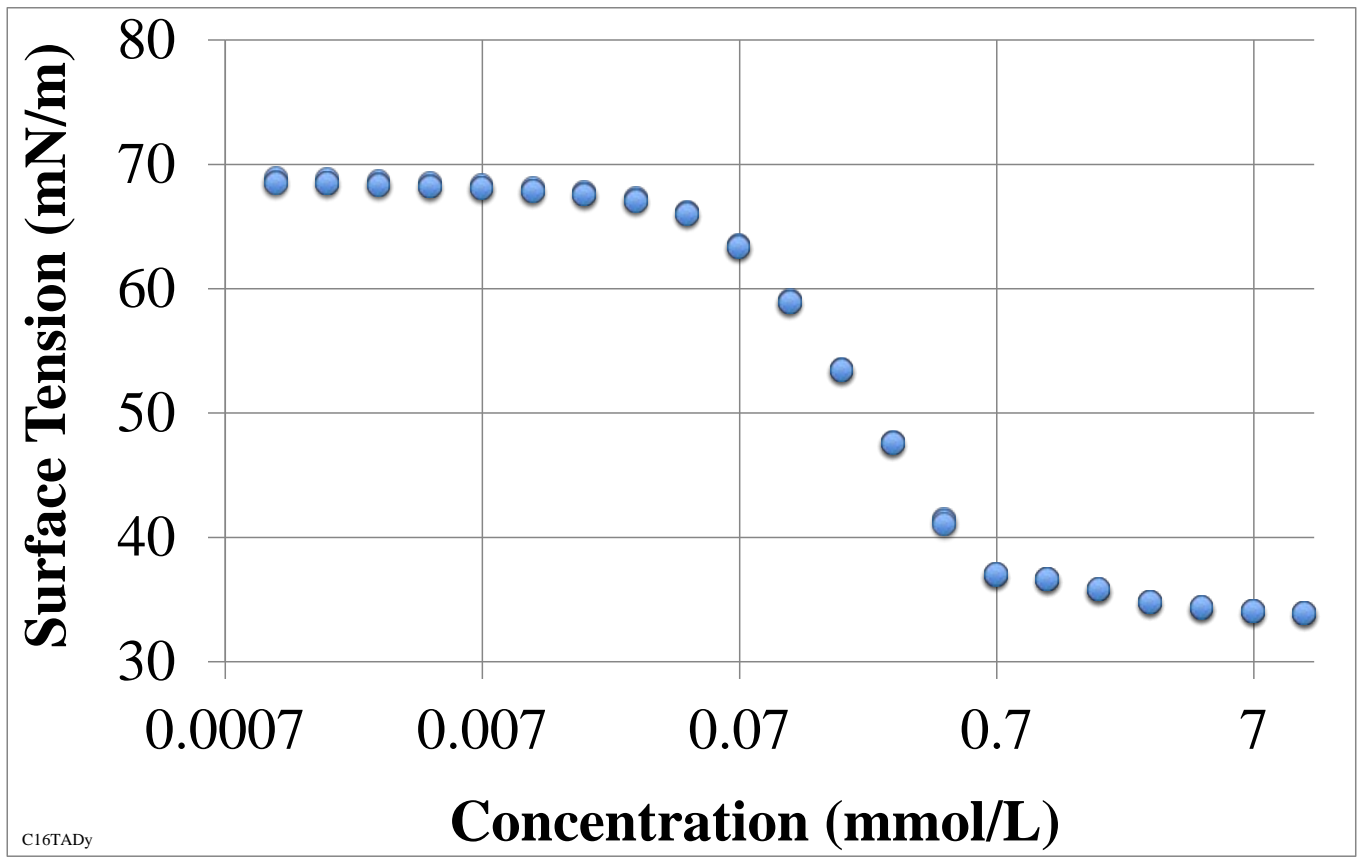

Figure S3. Tensiometer data for $\mathrm{C}_{16} \mathrm{TADy}$ used to determine the critical micelle concentration at $0.69 \mathrm{mmol} / \mathrm{L}$.

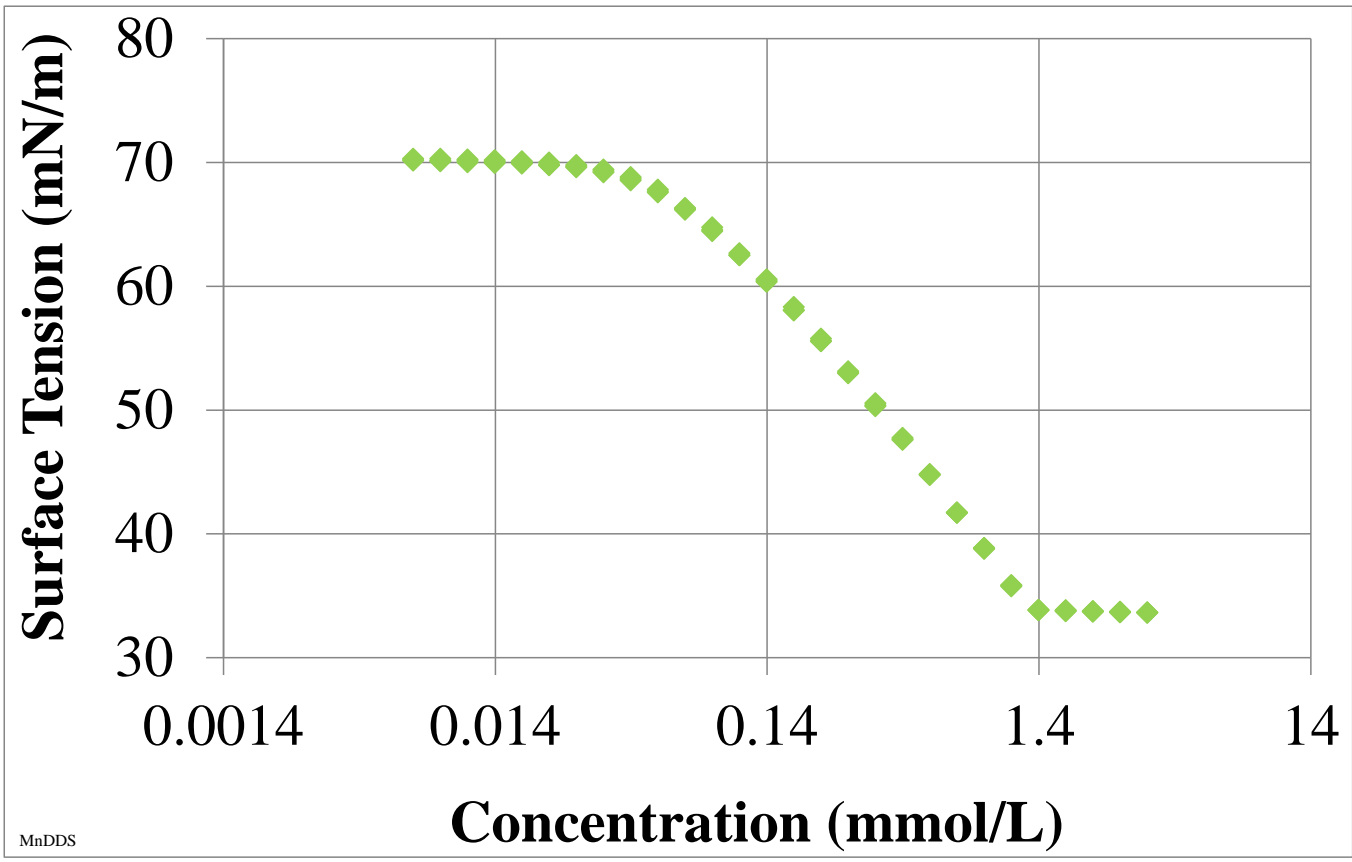

Figure S4. Tensiometer Data for MnDDS used to determine the critical micelle concentration at $1.4 \mathrm{mmol} / \mathrm{L}$. 
Table S1. Surface Tension of $\mathrm{C}_{16} \mathrm{TAFe}$ solutions inside of various uniform magnetic fields. Measurements are given in $\mathrm{mN} / \mathrm{m}$ and errors are reported as the standard error of the mean.

\begin{tabular}{|c|c|c|c|c|c|c|}
\hline \multirow{2}{*}{$\begin{array}{l}\mathrm{C}_{16} \mathrm{TAFe} \\
\begin{array}{c}\text { Concentration } \\
(\mathrm{mM})\end{array} \\
\end{array}$} & \multicolumn{6}{|c|}{ Field Strength (Tesla) } \\
\hline & 0.00 & 0.46 & 0.48 & 0.52 & 0.60 & 0.68 \\
\hline 0.00 & $72.3 \pm 0.2$ & $72.6 \pm 0.3$ & $72.7 \pm 0.2$ & $72.6 \pm 0.2$ & $72.8 \pm 0.3$ & $72.8 \pm 0.3$ \\
\hline 0.15 & $61.9 \pm 0.2$ & $61.9 \pm 0.2$ & $61.9 \pm 0.1$ & $61.6 \pm 0.1$ & $61.6 \pm 0.1$ & $61.1 \pm 0.1$ \\
\hline 0.31 & $52.8 \pm 0.2$ & $52.7 \pm 0.2$ & $52.3 \pm 0.1$ & $52.3 \pm 0.1$ & $52.4 \pm 0.1$ & $52.0 \pm 0.1$ \\
\hline 0.44 & $45.5 \pm 0.2$ & $45.6 \pm 0.1$ & $45.5 \pm 0.1$ & $45.2 \pm 0.2$ & $45.0 \pm 0.1$ & $44.8 \pm 0.2$ \\
\hline 0.57 & $39.6 \pm 0.2$ & $39.4 \pm 0.2$ & $39.3 \pm 0.2$ & $39.0 \pm 0.1$ & $39.0 \pm 0.1$ & $38.8 \pm 0.2$ \\
\hline 0.78 & $36.4 \pm 0.2$ & $36.0 \pm 0.2$ & $35.8 \pm 0.2$ & $35.9 \pm 0.1$ & $35.9 \pm 0.1$ & $35.8 \pm 0.1$ \\
\hline 1.00 & $34.8 \pm 0.1$ & $34.6 \pm 0.1$ & $34.6 \pm 0.1$ & $34.4 \pm 0.2$ & $34.3 \pm 0.1$ & $34.4 \pm 0.1$ \\
\hline 1.20 & $34.1 \pm 0.2$ & $33.6 \pm 0.1$ & $33.6 \pm 0.2$ & $33.5 \pm 0.1$ & $33.6 \pm 0.1$ & $33.5 \pm 0.2$ \\
\hline
\end{tabular}

Table S2. Surface tensions of MnDDS solutions inside of various uniform magnetic fields. Measurements are given in $\mathrm{mN} / \mathrm{m}$ and errors are reported as the standard error of the mean.

\begin{tabular}{|c|c|c|c|}
\cline { 2 - 4 } \multicolumn{1}{c|}{ MnDDS } & \multicolumn{3}{c|}{ Field Strength (Tesla) } \\
\hline $\begin{array}{c}\text { Concentration } \\
\text { (mM) }\end{array}$ & $\mathbf{0 . 0 0}$ & $\mathbf{0 . 4 6}$ & $\mathbf{0 . 6 8}$ \\
\hline $\mathbf{0 . 7 0}$ & $38.6 \pm 0.2$ & $37.4 \pm 0.4$ & $37.3 \pm 0.2$ \\
\hline $\mathbf{1 . 0 5}$ & $35.3 \pm 0.3$ & $34.8 \pm 0.2$ & $34.4 \pm 0.2$ \\
\hline $\mathbf{1 . 4 0}$ & $33.9 \pm 0.3$ & $32.6 \pm 0.2$ & $32.6 \pm 0.3$ \\
\hline $\mathbf{1 . 7 5}$ & $33.4 \pm 0.1$ & $32.3 \pm 0.2$ & $31.6 \pm 0.2$ \\
\hline $\mathbf{2 . 1 0}$ & $33.0 \pm 0.4$ & $31.0 \pm 0.2$ & $30.9 \pm 0.2$ \\
\hline
\end{tabular}


Table S3. Surface Tension of $\mathrm{C}_{16} \mathrm{TADy}$ solutions inside of no magnetic field, a uniform magnetic field, and a magnetic gradient. Measurements are given in $\mathrm{mN} / \mathrm{m}$ and errors are reported as the standard error of the mean.

\begin{tabular}{|c|c|c|c|}
\hline C16TADy & \multicolumn{3}{|c|}{ Field Strength (Tesla) } \\
\hline $\begin{array}{c}\text { Concentration } \\
\text { (mM) }\end{array}$ & $\mathbf{0 . 0 0}$ & $\begin{array}{c}\text { Uniform } \\
\mathbf{0 . 6 8}\end{array}$ & $\begin{array}{c}\text { Gradient } \\
\mathbf{0 . 2 7}\end{array}$ \\
\hline 0.15 & $60.5 \pm 0.1$ & $59.3 \pm 0.2$ & $46.8 \pm 0.9$ \\
\hline 0.31 & $47.4 \pm 0.1$ & $46.4 \pm 0.2$ & $35.9 \pm 1.7$ \\
\hline 0.44 & $40.8 \pm 0.1$ & $39.6 \pm 0.1$ & $31 \pm 2$ \\
\hline 0.57 & $37.1 \pm 0.1$ & $36.4 \pm 0.1$ & $31.7 \pm 1.3$ \\
\hline 0.78 & $35.4 \pm 0.1$ & $34.7 \pm 0.2$ & $31.3 \pm 0.9$ \\
\hline 1.00 & $34.5 \pm 0.1$ & $33.8 \pm 0.1$ & $27.8 \pm 0.7$ \\
\hline 1.20 & $34.3 \pm 0.1$ & $33.7 \pm 0.1$ & $28.0 \pm 0.8$ \\
\hline
\end{tabular}

